\title{
El swarming como modelo analítico para pensar las políticas educativas
}

César Tello

RESUMEN: En este trabajo se presentan algunas reflexiones de carácter teórico-analítico sobre el modelo del swarming como un modo posible, entre otros, para comprender el objeto de estudio de la política educacional, esto es: las políticas educacionales. Estas reflexiones se desarrollan en el marco de lo que hemos denominado el Campo de los estudios Epistemológicos en Política Educacional. Así, se propone un modelo teórico para el análisis de las políticas educacionales desde una perspectiva epistemológica pluralista. Realizando en primer término una caracterización de los modelos empleados para el análisis de las políticas públicas provenientes del desarrollo teórico de las ciencias políticas y concibiendo, desde nuestra perspectiva, a las políticas educacionales como multidimensionales, complejas y rizomáticas que se manifiestan modélicamente a través del swarming.

Palabras Clave: investigación; políticas educacionales; modelizaciones teóricas; swarming.

RESUMO: Este trabalho apresenta algumas reflexões da natureza teórico-analítica sobre o modelo do "swarming" como um modo possível, entre outros, para compreender o objeto de estudo da política educacional, isto é: as políticas educacionais. Estas reflexões são desenvolvidas no marco do que temos chamado o Campo de Estudos Epistemológicos em Política Educacional. Assim, é proposto um modelo teórico para a análise das políticas educacionais a partir de uma perspectiva epistemológica pluralista O artigo apresenta uma caracterização dos modelos utilizados para a análise das políticas públicas provenientes do desenvolvimento teórico da ciência política e concebe, a partir de nossa perspectiva, as políticas educacionais como multidimensionais, complexas e rizomáticas, que se manifestam em uma modelização através do "swarming".

PALAVRAS-CHAVE: pesquisa; políticas educacionais; modelizaçãos teoricas; swarming 


\section{Presentación}

En este artículo analizamos la política educacional como campo teórico en relación a los diversos modos de comprender su objeto estudio: las políticas educativas ${ }^{1}$. Para esto es necesario considerar que existen múltiples modelizaciones teóricas, en particular, desarrolladas desde las ciencias políticas en relación a como se establecen, despliegan e impactan - entre otros términos posibles ${ }^{2}$ - las políticas públicas.

En este sentido consideramos que no es casual la selección de un objeto de estudio en una investigación en políticas educacionales, sino que surge de un modelo previo del investigador. El modelo que el investigador posee sobre las políticas educacionales surge de un posicionamiento epistemológico (véase TELLO, 2012a, c; 2013a, b, c). Dependiendo de la concepción que tiene el investigador se inclinará por determinada construcción de su objeto de estudio para la investigación en política educacional. Las concepciones que posee el investigador en forma de modelizaciones teóricas devienen de la propia formación del investigador, de su construcción subjetiva y de las producciones teóricas con las que se ha vinculado ${ }^{3}$.

Ahora bien, es necesario considerar que las múltiples modelizaciones vienen de los trabajos que han plantado en su desarrollo histórico las ciencias políticas, a través de diversos referentes teóricos y que ninguna de ella posee un carácter neutral o totalmente explicativo y menos aún verdadero. Sin embargo, esas modelizaciones impactan sobre la construcción subjetiva en la formación del investigador en política educacional.

Estas modelizaciones se proponen explicar el surgimiento, el cambio o el desarrollo de las políticas públicas, comprendiendo que el trabajo científico consiste, en gran parte, en proponer teorías para explicar fenómenos, en nuestro caso específico de las políticas educacionales.

En este artículo desarrollamos algunas consideraciones teóricas que venimos reflexionando sobre lo que hemos denominado el Campo de los Estudios Epistemológicos en Política Educativa ${ }^{4}, \mathrm{y}$ en primer término caracterizamos algunas modelizaciones que se han venido desarrollando fuertemente en los ámbitos académicos para presentar finalmente el modelo del swarming que intenta acercarse a una concepción pluralista de la comprensión de las políticas educacionales, y que como otros modelos, en términos

\footnotetext{
${ }^{1}$ No tomamos aquí la compleja distinción anglosajona entre politics y policy. Dado que esta distinción es ambigua al intentar traducirla al español o al portugués. Como afirma Cox "no se puede recuperar el sentido del segundo término si no es especificando políticas sectoriales.” (2006, p. 3). Por esta razón tomamos la categoría de políticas educacionales (en plural) para referirnos a la acción política y la política educacional (en singular) para referirnos al campo teórico.

2 Aquí es necesario señalar que el empleo de un determinado término indica un posicionamiento epistemológico de comprender las políticas. No es lo mismo referirse al "desarrollo de las políticas", "impacto de políticas", "estructura de las políticas", etc. Cada una de estas expresiones, como veremos más adelante, posee una determinada mirada sobre la concepción de políticas.

${ }^{3}$ En este sentido es clave, para quienes nos desempeñamos como formadores de investigadores en política educacional, "mostrar" la mayor cantidad posible de modelos teóricos analíticos para la reflexión y análisis profundo de los estudiantes. En ocasiones puede suceder que los investigadores formados no estemos de acuerdo con algunos modelos, pero es necesario que los estudiantes los conozcan, estudien y reflexionen sobre ellos.

${ }^{4}$ Véase Tello, 2009; 2011; 2012a; 2012b; 2013a;2013b; 2013c; 2014a;2014b; Tello y Mainardes, 2012; Tello y Mainardes; 2014; Mainardes, 2009; Mainardes, 2013.
} 
de Jobert y Muller (1987), es una imagen de la realidad social, considerando que en la investigación en educación las imágenes nos permiten tener un poco más de claridad en las complejas relaciones sociales y políticas. O como plantean Muller y Surel (1998) es preciso señalar que la política pública no existe 'naturalmente' en la realidad, sino que se trata a la vez de una construcción social y de una construcción de un objeto de estudio. Esto implica dificultades para la identificación de sus contornos exactos, en este sentido las modelizaciones nos permiten llevar a cabo ejercicios heurísticos para comprender la hechura de la política educacional.

\section{Caracterizando algunos modelos teóricos de las políticas educacionales}

En esta sección presentamos algunos modelos teóricos ${ }^{5}$ que han contribuido y siguen contribuyendo a la comprensión de las políticas educacionales. Entendiendo que el campo teórico de la política educacional ha tomado varios de estos modelos de las ciencias políticas; el nacimiento de la ciencia política moderna se produce a través de la separación de los estudios políticos respecto de la matriz tradicional del derecho. En el año 1948 se marca el hito fundacional en la ciencia política: a pedido de la UNESCO, se reúnen en París expertos y estudiosos con la finalidad de tratar de redefinir y acotar su objeto de estudio. Las deliberaciones concluyen con la confección de la célebre "Lista Tipo", elaborada bajo una fuerte influencia del pensamiento anglosajón. Si bien ella constituye tan sólo una mera enumeración pragmática de temas, sigue siendo a pesar de los sesenta años de su elaboración un eje de referencia para la elaboración de marcos teóricos y la confección de currículas universitarias en relación a las ciencias políticas, incluyendo el área curricular de la política educacional actual en las carreras de pedagogía y ciencias de la educación. Este no es un es dato menor y menos aún un buen signo para los investigadores en ciencias políticas en general y en política educacional en particular. Ya que siguiendo los postulados de Giovanni Sartori (2004) en un reconocido artículo denominado “¿Dónde está la ciencia Política?”, César Cansino en el año 2006 (y luego en el 2008) anuncia el adiós a la ciencia como la crónica de una muerte anunciada con muchos debates a su alrededor. Explicando que "terminó alejándose del pensamiento y la reflexión, hasta hacer de esta ciencia un elefante blanco gigantesco,

\footnotetext{
5 Debemos considerar que aquí solo se presentan algunos modelos analíticos. No ingresamos en el desarrollo de enfoques y perspectivas teóricas como el institucionalismo, neo institucionalismo etc. Entendiendo de este modo una clara diferenciación entre 'modelos analíticos' y enfoques, perspectivas, posicionamientos o metodologías de investigación. En muchas ocasiones se confunde la categoría de modelos analíticos con metodologías o lo que hemos denominado en otro lado 'epistemetedología'(TELLO, 2012; 2013)

${ }^{6}$ Los distintos temas propuestos quedan agrupados en cuatro secciones: I. Teoría política: a) Teoría política. b) Historia de las ideas políticas. II. Instituciones políticas: a) Constitución. b) Gobierno central. c) Gobierno regional y local. d) Administración pública. e) Funciones económicas y sociales del gobierno. f) Instituciones políticas comparadas. III. Partidos, grupos y opinión pública: a) Partido político. b) Grupos y asociaciones. c) Participación del ciudadano en el Gobierno y la Administración. d) Opinión pública. IV. Relaciones internacionales: a) Política internacional. b) Organización y administración internacional. (UNESCO 1948).
} 
repleto de datos, pero sin ideas, ni sustancia, atrapada en saberes inútiles para aproximarse a la complejidad del mundo." (p. 13) y de algún modo la investigación en políticas educacionales vive esta misma crisis epistémica. En el mismo eje de reflexión De Sierra et. al. (2006, p. 51) señalan que en América Latina en los últimos años se debilita en las ciencias sociales la dimensión critico-utópica afirmando que "hay menos reflexión sobre la disciplina y más sobre el uso que la sociedad hace del conocimiento de las ciencias sociales".

En esta línea de análisis, debemos considerar que dotada de las características principales de las ciencias políticas, surgía en la década de $1950^{7}$ la política educacional como campo teórico, con una visión fuertemente centrada en la legislación y en algunos casos en educación comparada. Esta última no poseía el desarrollo epistemológico actual sino que, básicamente consistía en establecer ejes de comparación legislativos de diversos países y de la estructura del sistema educativo, entre otros temas de comparación lineal y casi descontextualizada. Pero sin duda respondía a la matriz analítica de las ciencias políticas en Latinoamérica con el enfoque jurídico-institucionalista.

$\mathrm{Y}$, al referirnos a esta cuestión es ineludible analizar algunos de estos modelos, y comenzar con quien fuera el creador del enfoque de políticas públicas a través del modelo de análisis de políticas públicas: Harold Lasswell. Los modelos para el análisis de las políticas educacionales, devienen de las reflexiones del origen del estudio de las políticas públicas, desde Laswell (1951) con su perspectiva pragmática y el clásico modelo de fases sucesivas al que llamó proceso de decisión conformado por siete pasos: 1-Inteligencia 2-Promoción 3-Prescripción 4-Invocación 5-Aplicación 6-Terminación 7-Evaluación, del cual se deprenden luego otras etapas diseñadas como modelo de interpretación. Entre las más conocidas se encuentran la de Brewer (1974), quien fuera alumno de Laswell, y agrupaba los siete pasos como: a-agenda, b-formulación, c-implementación y d-evaluación, en términos de modelos input-output como guía epistemológica. O los ya clásicos pasos de Meny y Thoenig (1992): a)La identificación de un problema b)La formulación de soluciones c)La toma de decisión d)La ejecución del programa e)La terminación de la acción.

El modelo de Laswell inició un nuevo modo de pensar los procesos políticos, pero por otro lado instaló la idea de las etapas para el estudio de las políticas públicas muy arraigado hasta nuestros días. Del mismo modo, este modelo teórico, instaló la idea de los especialistas en etapas, fuertemente arraigada en la investigación en políticas educacionales y esto se puede ver en la actualidad con investigadores que estudian procesos de implementación o agendas o evaluación de políticas educacionales. Y esto, en ocasiones, genera un mirada reduccionista de la investigación de los procesos políticos.

Sin duda, que el surgimiento no puede ubicarse precisamente. Encontramos en el relevamiento empírico algunos casos de cátedras o espacios curriculares de política y educación a principio de 1940, en México. Sin embargo, fueron reemplazados por otras asignaturas y volvieron a emerger en la siguiente década. 
Este modelo y sus múltiples variaciones devienen del enfoque institucionalista que supone que las políticas públicas son las actuaciones del Estado. Así Deubel (2008) explica que existe cierta centralidad en los estudios en políticas públicas, desde una modelización como el public choice, que según el autor "se centra en analizar los arreglos institucionales, en particular el diseño de las instituciones estatales y las normas legales y constitucionales" (p. 80).

También desde esta modelización se suele considerar que lo público es lo estatal y que todas las políticas son públicas. Tal enfoque supone erróneamente que las políticas públicas son siempre explícitas y neutrales y en ocasiones confunde Estado con gobierno.

Por otro lado es necesario presentar el denominado modelo teórico de la interacción política cuyo principal exponente se centra en la figura de Lindblom que cuestionó el énfasis en el racionalismo de Laswell a través de su libro "La ciencia de salir del paso" del año 1959, empleando en su análisis de la acción pública un modelo propio que denominó de la decisión incremental. Cuyo principal fundamento es que la toma de decisiones no es racional, por el contrario las políticas se van desplegando por decisiones instintivas y empíricas de los administradores de la burocracia política, lejanos a la tomas de decisión racional. Entendiendo que el decisor debe "salir del paso", considerando que son clave en este proceso otras variables de análisis tales como las relaciones de poder, y de la integración entre las diversas fases del proceso decisorio, qué no tendrían necesariamente un fin o un principio (RUIZ VIÑALS, 2004).

El modelo de Lindblom asume que los procesos de las políticas suceden en fases (formulación de la política, decisión e implementación), basado en una visión en la que el tomador de decisiones primero analiza el problema y las alternativas y luego toma una decisión acerca de cuál opción deberá escoger.

Otros de los modelos es la teoría sistémica de David Easton y su concepción de funcionamiento del sistema político que emplea el modelo de sistema utilizado en las ciencias biológicas (la metáfora de la célula). Concibe un sistema político (caja negra) que recibe insumos o demandas (inputs) y los transforma en productos -leyes, decisiones, o políticas públicas- (outputs). Estos productos tienen un impacto sobre la sociedad política (la opinión pública, la realidad, etc.) que es reenviado hacia el sistema político como retroalimentación (feedback), y así el ciclo político recomienza. Sin dudas que Easton se basó en la teoría de los sistemas de Bertalanffi.

Con su teoría, Easton inquiere construir un esquema general unificado que permita un análisis uniforme y semejante de la vida política en sus variadas manifestaciones, al igual que Laswell y Lidblom, se interesa especialmente por la estabilidad y el orden, por los mecanismos que posibilitan la persistencia de los sistemas políticos en un mundo de cambios y tensiones.

También es necesario reseñar el modelo de los ciclos de Jones que para la linealidad analítica de los modelos previos desplegó el modelo del policy cycle (JONES, 1970) que 
posee la ventaja de focalización sobre los procesos más que sobre las instituciones, de todos modos el modelo no hace otra cosa desagregar o segmentar la política pública con una tendencia a sesgar el análisis y en general se sitúa la implementación y evaluación solo al final de los ciclos.

Estos modelos poseen un fuerte componente de pasos y etapas. Y, por ejemplo Muller (1998) formula una serie de críticas a estos enfoques, la primera en relación al orden lineal que presentan, al respecto afirma que "El orden de las etapas puede ser inverso o perturbado" (p. 67), así también, sostiene que en algunos casos es muy difícil identificar las etapas debido a que no es siempre es fácil delimitar cada una.

También debemos señalar el modelo político de las redes y de las comunidades (KRIESI, 1994; SABATIER Y JENKINS-SMITH, 1988). En términos de Sabatier y Jenkins-Smith (1988) se desplegaron estos modelos con la categoría de las advocacy coalitions a partir de la cual se intentó iniciar la búsqueda de una síntesis de los mejores dispositivos aportados por los enfoques top down y bottom up en el estudio de las políticas públicas. Deubel (2007), plantea que esta concepción considera "las interacciones entre sociedad y Estado, más en sus dimensiones horizontales (el uno y el otro son socios) que verticales (el uno domina al otro o viceversa) y de señalar la interpenetración creciente entre las esferas pública y privada" (p. 51).

Otro modelo analítico es el del abordaje cognitivo de políticas públicas (MULLER, 1990, 2000; MULLER; SUREL, 2002) a partir de la cual se plantea que cada política representa una tentativa de resolver un problema de la sociedad, configurada a partir de procesos de decisión en la cual participa un cierto número de actores. La política pública y la expresión de poder público construyen un cuadro normativo de acción constituyendo un determinado orden local. Así, el análisis cognitivo de las políticas requiere estudiar el papel de las ideas desarrolladas por los actores, las ideas en acción, el referencial global y local que fundamentan la política y los mediadores que intervienen en esas políticas.

Estos modelos se caracterizan básicamente por poseer claridad en quienes son los actores de un proceso de política: formuladores, coordinadores, evaluadores y rendidores de cuentas de las políticas públicas, aplicadores

Por ejemplo desde una mirada de top down o bottom up implica reconocer el comienzo y el final en un análisis vertical, casi positivista. Y si pensamos las políticas educacionales como movimiento horizontal, en un continuo de decisiones?. Las cuales se van transformando a cada paso, de forma progresiva: autoridades de un gobierno estatal, maestros, padres, en donde la toma de decisiones despliega transformaciones y más transformaciones en el desarrollo des-ordenado y des-plegado de la acción política. Donde unas implican y transforman a las otras?.

Básicamente estos son los modelos analíticos en la investigación en políticas públicas, con sus diversas variaciones y matices. Y, los que se emplean en la investigación en política educativa en la actualidad, en ocasiones sin conocer estos referenciales teóricos, 
pero de algún modo los investigadores jóvenes o en formación poseen en sus matrices subjetivas alguna de estas miradas.

Argumentamos que estos modelos de análisis de las políticas públicas siguen sumidos en el modelo de la secuencialidad, bajo el esquema del proceso de las políticas públicas (etapas o fases funcionales presentes en la vida de las decisiones públicas: iniciación, estimación, selección, implementación, evaluación, terminación) que no hacen más que continuar con la incapacidad de derrotar la linealidad positivista en la investigación en política educacional.

\section{El modelo pluralista del swarming para el análisis de políticas educacionales}

Partimos de una concepción pluralista en la que entendemos las políticas públicas como un continuo de decisiones, que comprende momentos y asuntos que se inter-vinculan en un flujo de interacciones entre los sujetos que hacen la política, interacciones que no siempre hacen referencia a una secuencia lógica de fases sino a un proceso dinámico y complejo, y en ocasiones con sorprendentes esquemas des-secuenciados, rizomáticos y de flujos continuos.

Con esto no estamos diciendo que no existan momentos, etapas o fases. Pero esas etapas siempre son vistas desde determinado ángulo y pueden ser no-secuanciales. $\mathrm{Si}$ consideramos que las políticas se implementan lo hacemos con una concepción de determinado modelo político.

En los modelos caracterizados previamente el Estado cumplo un rol central, casi causal. Aunque en otros modelos se incluyen otros componentes. Y, sin dudas, es allí donde se puede observar una concepción desde una determinada perspectiva y posicionamiento epistemológico del objeto de estudio de la política educacional.

Por esta razón debemos considerar al menos brevemente algunos debates que se dan en torno a la definición del objeto de estudio de la política educacional. Que, como en los modelos, también, deviene de los debates de las ciencias políticas.

El objeto de estudio de la política educacional es, como dijimos, las políticas educacionales en tanto la propia realidad socio-educacional, en sus múltiples dimensiones; consideramos que los diversos enfoques epistémicos generan en sí, un objeto en permanente construcción (GALINDO CÁCERES, 1999). Esto es, los ángulos de análisis de la realidad socio-educacional le permiten al campo teórico de la política educacional la construcción desde diversos ejes que confluirán en su objeto, por ejemplo el estado, la jurisprudencia, la micropolítica, el discurso político, los debates políticos, la gobernabilidad educacional, el derecho a la educación, entre otros.

Burch (2009), oponiéndose a la definición clásica del objeto de estudio de la política educacional como acción de un estado, explica que cada vez se hace más difícil sostener el modelo tradicional del objeto de estudio de la política educacional dado que el 
campo está cada vez más congestionado: nuevas relaciones y nuevas formas de relación están siendo establecidas en la política y en relación con ella. Y afirma que "los límites entre Estado, economía y sociedad civil son cada vez más imprecisos" explicando que existe una multiplicidad de voces que intervienen en los diálogos en torno a la política y nuevos conductos por los que se incorpora el discurso al debate de ideas sobre el tema. Ball (2011) afirma "Hoy en día, la política educacional y social dentro del gobierno se piensa, se hace y recibe influencias en muchos sitios diferentes, y la comunidad que constituye la politica educacional es cada vez más diversa e inestable" (p. 11, el énfasis es mío) lo cual implica un pasaje del desarrollo de "relaciones que suponen reciprocidad e interdependencia en contraposición a jerarquía e independencia" (PETERSON, 2003, p. 1. Citado en BALL, 2011). Con esto decimos que claramente existen métodos de análisis de las políticas educacionales centradas en el Estado, sin embargo al encontrarnos con una nueva configuración estatal esos métodos o abordajes de análisis, ni siquiera pueden ser los "clásicos", habrá que pensar nuevos esquemas analíticos para el análisis centrado en el Estado. Aún así, como explicamos más adelante el Estado no puede ser el centro de la política educacional en términos de investigación, sino una mediación para la investigación.

Teniendo en cuenta esta consideración podemos cuestionar de algún modo la caracterización que comúnmente se hace sobre el objeto de estudio de la política educacional como la acción de un Estado en relación al ámbito educativo. Y, nos preguntamos: ¿Qué sucede cuando la categoría "acción del estado" es muy difusa?. ¿Qué sucede cuando el objeto de estudio de la política educacional se comprende desde una perspectiva y posicionamiento epistemológico específico? ¿Y si se acuerda con politólogo italiano Paolo Virno? que afirma:

\footnotetext{
"El éxodo no es nostalgia, pero considerar al Estado-nación como un refugio sí es nostálgico. El éxodo no es un retroceder, sino un salir de la tierra del faraón; la tierra del faraón fue hasta hace una o dos generaciones el Estado-nación, los Estados nacionales son como caparazones vacíos, como cajas vacías y, por eso, sobre ellos se hace una carga emotiva que, naturalmente, es muy peligrosa, porque corre el riesgo de transformarse antes o después en xenofobia o, de todas maneras, en una actitud rabiosa y subalterna al mismo tiempo: rabia y subalternidad juntas, base de los distintos fascismos postmodernos." (VIRNO, 2006, p. 64).
}

Desde nuestra perspectiva, y como un modelo posible, entre otros, comprendemos la realidad de las políticas educacionales como sawrming donde el estado jugará un papel de mayor o menor preponderancia según el lugar y el ángulo de estudio del investigador, es decir: el posicionamiento epistemológico del investigador (véase TELLO 2012a, b; 2013a).

Entendemos las políticas educacionales como swarming en tanto trayecto intertextual. Stephen Ball (1989) ha definido las políticas educacionales como textos y como discursos, al referirse a las políticas educacionales como texto explica el autor (BALL, 
1989) que es el producto del trayecto sinuoso e imprevisto, que se genera en las distintas arenas políticas de cada contexto, mediante luchas, compromisos, interpretaciones y reinterpretaciones que luego serán decodificadas por los actores que aportarán diversos sentidos, elaborando ajustes secundarios al propio contexto de modo complejo.

En fin el objeto de estudio de la política educacional es el estudio de los procesos de la toma de decisiones en el swarming, donde existen múltiples componentes de análisis. Y el análisis desde una perspectiva estatal es un modelo y ángulo para la construcción de un objeto de estudio en política educacional, como hemos visto en los modelos reseñados en la sección anterior.

Dependerá del ángulo de estudio del investigador, y, aceptando esta premisa, varían los objetos de estudio que construye el investigador y por tanto sus resultados de investigación. En este sentido es clave considerar los resultados de investigación como resultados obtenidos desde una perspectiva: la del investigador.

Así, argumentamos que la clásica definición de la acción del Estado como objeto de estudio de las políticas educacionales es parte de la matriz histórica del surgimiento del campo teórico de la política educacional en una perspectiva jurídico-institucional influenciada por las ciencias políticas en su enfoque de políticas públicas desde Lasswell en adelante. Aproximándonos a reflexiones de la sociología de la política educacional como paradigma de análisis podemos ingresar en otros ángulos de análisis.

El swarming posee mayor complejidad que los ciclos o las políticas entendidas como red, y sin duda mayor dinámica que los modelos lineales, porque:

1. se asume el movimiento de la política educacional. Y que el proceso de investigación no es más que una fotografía de ese movimiento.

2. se requiere mutilar el swarming para estudiar la política educacional y simultáneamente tener una comprensión general de él, sino se convierte en incomprensible el objeto de estudio de la investigación.

3. las posiciones de poder no son fijas, dependerá del ángulo recortado

4. No existe un arriba y abajo un dominante y un dominado a priori. Quizá puede establecerse a posteriori de la investigación.

Bowe et. al. (1992) advierten sobre la dificultad de comprender y analizar las políticas educacionales de un modo verticalista. Del mismo Raab (1994) explica que el Estado debe comprenderse como un componente más de la compleja trama de las políticas educacionales. En este sentido al reflexionar sobre el referencial teórico de Sabatier, Deubel (2008) plantea que: "la unidad de análisis no puede limitarse a la estructura gubernamental, sino a un "subsistema de política". Este subsistema, como parte del sistema político, está compuesto por una variedad de actores, públicos y privados, que están activamente implicados o interesados en un problema de política o en una controversia" (DEUBEL, 2008, p. 82). 
Y siguiendo nuestros postulados, donde el ángulo de estudio, es decir: el objeto de estudio, puede ser o no el estado. Debemos considerar que en las investigaciones sobre política educacional existe un debate internacional a este respecto, por caso, y solo como un ejemplo entre otros, señalamos las críticas de Ozga a una investigación desarrollada en Gran Bretaña y de la cual indica que se pueden sacar pocas conclusiones porque a esa investigación le faltó un abordaje de las teorías centrada en el Estado (véase POWER, 2006). Ozga, explica que "las teorías centradas en el estado son capaces de proveer explicaciones teóricamente más adecuadas y coherente sobre la textura y la dirección de los cambios educacionales" (POWER, 2006, p. 14). Por otro lado, varios autores del campo de las políticas educacionales plantean que una teoría centrada en el Estado no logra explicar las transformaciones de las políticas educacionales, aquí se sitúan varios investigadores en políticas educacionales, particularmente de Gran Bretaña, que es donde con mayor énfasis se ha dado este debate ${ }^{8}$ : Ball (1990; 2011); Bowe et. al. (1992); Raab (1994); Du Gay (2000); Power (2006); Burch (2009); entre otros. Y, otros investigadores que consideran que sin un abordaje de una teoría centrada en el Estado se hace imposible comprender el desarrollo de las políticas educacionales, como los casos de Dale (1989); Gordon (1989); Ozga (1993); Pannu (1996), Robertson (2011), entre otros.

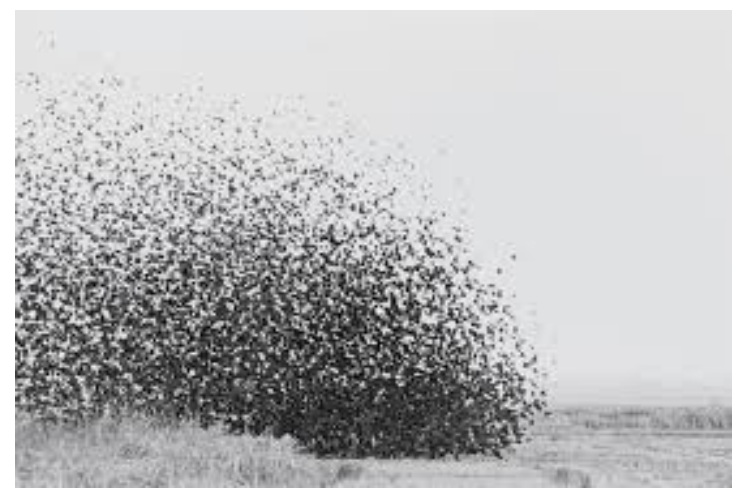

Así es que definimos la compleja movilidad de las políticas educacionales en términos de swarming. Esta categoría no tiene una traducción clara al español ni al portugés, un swarm es un enjambre de abejas y el swarming son las abejas en movimiento (enjambramiento) en búsqueda de un sitio donde construir el panal. Son las abejas en movimiento, pero juntas, casi sin poder identificar la tradicional jerarquía que estas tienen, dado que la búsqueda del sito para la construcción del panal no está circunscripta a la decisión de la Abeja Reina. De algún modo el modelo del swarming se opone a los modelos clásicos del pensamiento moderno, dado que nuestro modelo no intenta ser utópico, por el contrario asumimos las perspectiva de Foucault (1986) en términos de un modelo que se caracteriza por la heterotopía, que en contraste con el espacio utópico, las heterotopias se definen por "ser capaces de yuxtaponer en un único espacio real varios

\footnotetext{
${ }^{8}$ Es importante considerar el debate sobre esta cuestión producido en otras partes del mundo, porque en muchas ocasiones se emplean estos referentes teóricos sin conocer cuáles son las reflexiones profundas y las perspectivas que asumen en cuanto al rol del Estado en la investigación en políticas educacionales
} 
espacios, varios sitios [...] la época del cerca y el lejos, del lado a lado, de lo disperso". (p. 25). Los límites del swarming se tornan difusos en sus contornos, las abejas dentro del swarming se encuentran en constante movimiento, interactúan, van intercambiando posiciones pero sin forma ordenada.

En este sentido las políticas educacionales como acción política poseen múltiples actores, vinculaciones, decisiones, luchas de poder y acciones. Las preguntas clave aquí $\mathrm{y}$, tomando los modelos anteriores en relación al modelo pluralista del swarming son: ¿se pueden definir y conceptualizar las políticas educacionales? ¿Cuántos miles de abejas se observan en la fotografía? ¿Cuántos componentes teóricos, actores, instituciones, decisiones, implica las políticas educacionales como swarming?

Mi argumento es que una respuesta a estos interrogantes requeriría de varias enciclopedias que creo que nunca se terminarían de escribir. Ahora bien, sí se puede hacer un recorte epistemetedológico (véase TELLO, 2012b, c; 2013a,c) a los fines de construir un objeto de estudio, claramente artificial y desde un posicionamiento epistemológico. Esto significa, construir un objeto de estudio para investigar en políticas educacionales implica reconocer el recorte del swarming y simultáneamente no desconocer, al menos, los principales rasgos del swarming en su conjunto. Y comprender que ese objeto ya no estará más en movimiento al momento de definirlo como objeto de estudio. También se debe tener en cuenta que los modelos analíticos para el análisis de políticas educacionales no deberían ser determinantes. Por ejemplo, ¿dónde comienzan las políticas educacionales? o ¿dónde culminan?, sin dudas que intentar responder esta pregunta es asumir una posición y una perspectiva epistemológica, tómese por caso el modelo que tomamos de Brewer o de Easton donde claramente se asume una visión institucionalista del análisis de políticas.

En la propuesta de la política educacional del swarming la asumimos como una intertextualidad, como swarming, los textos están en constante transformación en la acción política. Es aquí donde en general se produce la confusión de la trayectoria política, esto es: entre quienes tienen mayor o menor poder en las acciones de gobierno. Con esto decimos, se producen luchas de poder, pero no necesariamente las ganan quienes tienen mayor poder. El proceso es mucho más complejo dado que las implicancias recíprocas entre los actores con distintos grados de poder transforma el poder del otro en una perspectiva intertextual y rizomática. En texto reciente (TELLO; MAINARDES, 2014) hemos definido algunas características de la perspectiva epistemológica pluralista y en la cual se encuadra el modelo del swarming. Allí planteamos que para Deleuze, un rizoma es un modelo descriptivo en el que la organización de los elementos no sigue líneas de subordinación jerárquica -con una base o raíz, dando origen a múltiples ramas, de acuerdo al conocido modelo del árbol de Porfirio-, sino que cualquier elemento puede afectar o incidir en cualquier otro. En un modelo arbóreo o jerárquico tradicional de organización como las taxonomías del top down, el bottom up o el policy cycles lo que 
se afirma de los elementos de mayor nivel es necesariamente verdadero de los elementos subordinados, y también puede suceder a la inversa.

En cambio en un modelo rizomático, cualquier predicado afirmado de un elemento puede incidir en la concepción de otros elementos de la estructura, sin importar su posición recíproca. El rizoma carece, por lo tanto, de centro.

En fin, la idea que queremos plantear sobre las políticas educacionales en términos de acciones políticas es que conforman una intertextualidad rizomática a modo de swarming.

No consideramos la existencia de las políticas educacionales como swarm, dado que la acción política por propia definición siempre se encuentra en movimiento. Movimiento de programas políticos, movimiento subjetivo en los actores que conforman y transforman la acción política.

En Tello y Mainardes (2014) hemos señalado que:

La perspectiva epistemológica del pluralismo se refiere a distintas posiciones relacionadas entre sí; el concepto de pluralismo indica la creencia que la realidad consiste de muchas "sustancias" distintas, en contraste con los conceptos monista y dualista.

En la epistemología, el pluralismo mantiene la idea que hay distintas, conflictivas, pero al mismo tiempo, verdaderas descripciones del mundo. Así, el swarming como modelo teórico-analítico plantea la necesidad de comprender la multidimensionalidad de las políticas educacionales. Y es desde allí que puede indagarse la realidad política. Desde las dimensiones de análisis que no son otra cosas que las mediaciones que nos permiten indagar la realidad.

\section{Las mediaciones de estudio de la politica educacional en el modelo del swarming}

Como un modo de aproximación general que englobe las diversas multiplicidades para la caracterización del objeto de estudio de la política educacional en términos del swarming es necesario aclarar el planteo que venimos señalando en este trabajo. Nos referimos a la distinción entre objeto de estudio y mediaciones.

Claramente hemos planteado que comprendemos el objeto de estudio de la política educacional a las políticas educacionales y que, a las políticas educacionales las hemos modelizado en este trabajo como swarming. Ahora bien, dentro del swarming existen múltiples componentes que nos permiten abordarlo. Esos componentes son los que denominamos mediaciones y que cada investigador podrá centrar como eje de su objeto de estudio. Las mediaciones pueden ser: actores, sujetos, estado, gobierno, jurisprudencia, escuela, tomadores de decisiones, gobernantes, legisladores, maestros, directores, alumnos, entre otros.

En el swarming un investigador identifica la mediación, es decir, aquello que le permite estudiar una parte de las políticas educacionales, esto es: una parte del swarming. Ese es su objeto de estudio, su ángulo de análisis de las políticas educacionales. 
Como dijimos, tendrá su ángulo, pero no podrá desconocer el escenario que brinda el swarming, entre los cuales, claramente está el estado. Pero podrá tener mayor o menor preponderancia en su investigación según su ángulo.

Sin embargo, podrá decirse que esas mediaciones pueden ser válidas para otros campos. Y eso es correcto, también se puede emplear para otros campos como la pedagogía o estudios sobre el curriculum, entre otros.. Ahora bien, existe una particularidad: las políticas educacionales se centran en lo político. Desde nuestra perspectiva lo político, en tanto sucesos, fenómenos, acontecimientos y procesos es la toma de decisiones en educación. En cualquier ámbito y estructura educativa.

A los efectos de la organización del campo se hace necesario establecer al menos, en términos de episteme de época, que existen ciertos límites, claro está: nunca fijos, sino porosos que de algún modo y a partir de los otros campos de estudio establecen un espacio de estudio del campo de la política educacional, y como un modo, claramente artificial de acordar sobre qué hablamos y estudiamos, y que, en términos de organización del conocimiento resulta imprescindible para nombrar. Así el campo teórico de la política educacional se puede organizar en estudios sobre a) políticas docentes, b) políticas de financiamiento educativo c)políticas de desarrollo curricular d)políticas de gobierno del sistema educativo e)políticas de reforma educacional f)políticas de evaluación educacional g)Políticas universitarias h) Estudios de micropolítica educacional i) estudios de macropolítica educacional (centradas en el estado) j)Política y legislación del sistema educativo j)Política educacional comparada.

Siempre y cuando en estos estudios se cumplan con los requisitos mencionados anteriormente: la toma de decisiones en educación con un componente político y con las presencia de las mediaciones propias del campo.

Entendiendo, finalmente, que esto no es más que una construcción arbitraria y organizada por quienes constituimos el campo como investigadores. 
Ahora bien, continuemos con la cuestión de la organización en relación a la investigación en política educacional. Dentro del swarming se dan las mediaciones que, como dijimos, se convierten en los ejes de los objetos de estudio y desde allí los "estudios sobre", gráficamente lo podemos señalar del siguiente modo:

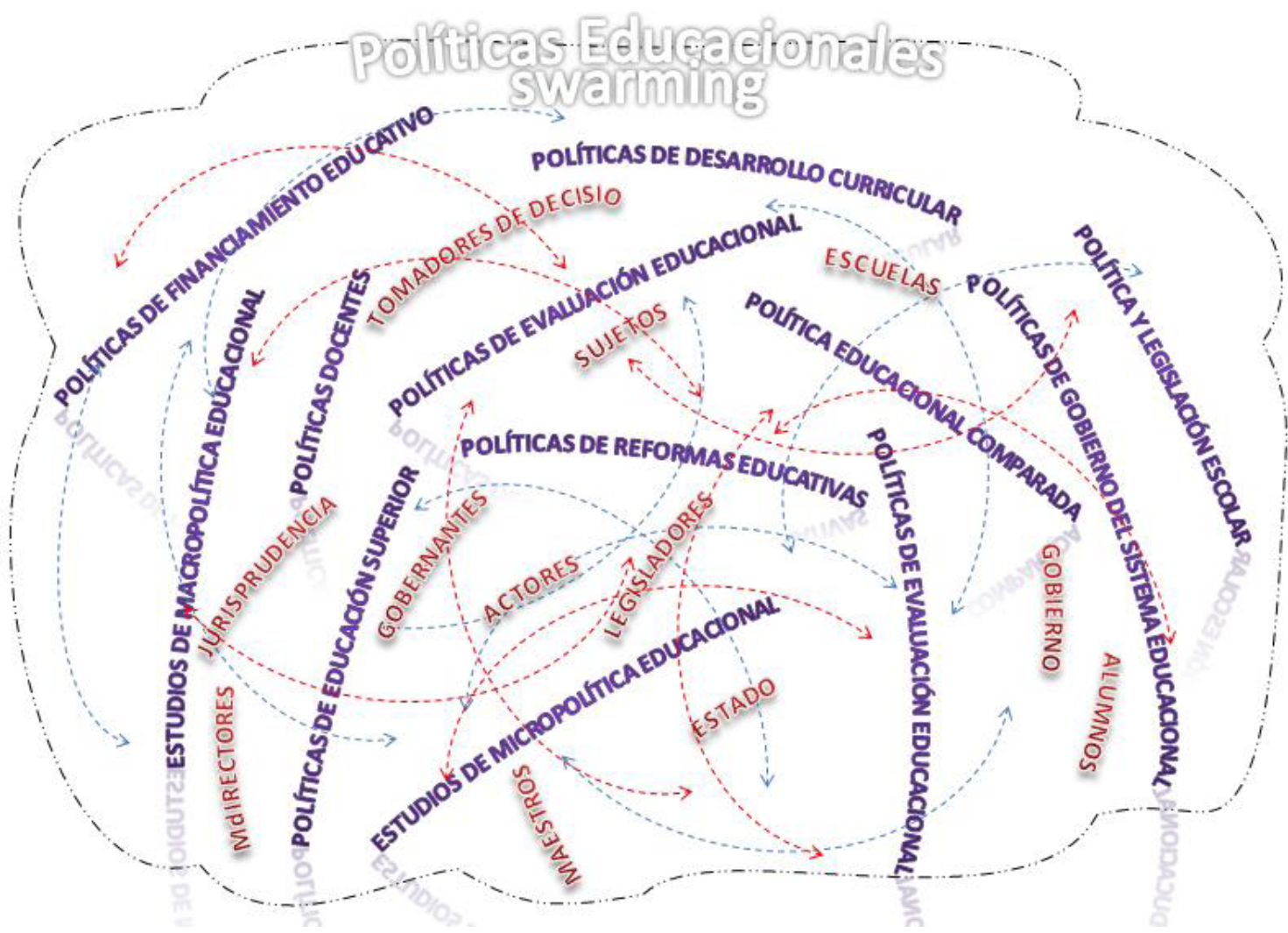

Figura 1: Las políticas educacionales como swarming.

Este es la representación del enjambramiento de las políticas educacionales con "los estudios sobre" señalados en azul y sus respectivas flechas de vinculación y las mediaciones y las fechas en rojo. Tanto las mediaciones como "los estudios sobre" se vinculan, interaccionan y des-pliegan, considerando que los colores se emplean al solo efecto de la identificación. Pero son múltiples las interacciones, las posibilidades, los ejes posibles de objetos de estudio.

Así, si la investigación se ha desarrollado cuidadosamente con categorías teóricas sólidas desde un posicionamiento epistemológico y asumiendo una perspectiva epistemológica se podrá decir con Pascal que "Lo contrario de una verdad no es el error, sino una verdad contraria". Bohr traduce este pensamiento de otro modo: "lo contrario de una verdad trivial es un error estúpido, pero lo contrario de una verdad profunda es siempre otra verdad profunda". 


\section{Notas finales}

De algún modo el modelo de análisis para la investigación en políticas educacionales que hemos presentado trata de alejarse de los enfoques centrados en los pasos sucesivos y la linealidad, buscando mayor complejidad en la comprensión de las políticas educacionales que son objeto de estudio del campo teórico de la política educacional.

Así en el modelo del swarming las jerarquías reales no siempre son las que formalmente están establecidas. Cada política, por su singularidad, tendrá un flujo distinto de actores que el analista tratará de descubrir y describir, sin perder de vista el escenario, pero tomando solo una parte de él.

Ante el posible cuestionamiento de presentar un esquema con excesivo relativismo epistemológico planteo que un esquema debe ser lo suficientemente flexible para que el investigador le de consistencia y dirección. Y las modelizaciones son solo eso: esquemas analíticos que deben sostenerse con contenido y sustancia. Pero el modelo en sí, es un modelo vacío.

Cabe señalar en esta presentación que ante el escenario de investigación en ciencias sociales y ciencias políticas, y en específico de las políticas educacionales asumimos los postulados de Wallerstein (1999) cuando plantea que las ciencias sociales tiene grandes limitaciones en el estudio de las realidades sociales por lo cual habría que pensar nuevos fundamentos epistemológicos, para esto propone un desafío: "impensar las ciencias sociales" que se opone a la categoría de re pensarla, dado que re pensarlas se convertiría en una barrera epistémica para la creación de nuevas epistemologías. Así comienzan a emerger -a nivel internacional y latinoamericano- algunas perspectivas analíticas de la realidad social como "pensamiento alternativo" (VERGARA ESTÉVEZ, 1991). Por su parte, Atilio Borón advierte que es necesario darse "una minuciosa discusión sobre algunos de los principales problemas que plantea la necesaria reconstrucción de las ciencias sociales sobre nuevas bases epistemológicas." (2006, p. 16).

La realidad de las ciencias sociales y políticas y en particular de la política educacional requiere una transformación epistemológica que permita construir y desplegar nuevas epistemologías para repensarla, a partir del atrevimiento científico que, de algún modo, resquebraje y sacuda las perspectivas epistemológicas actuales, lo cual permitirá - a partir de esa deconstrucción - un espacio para iniciar un nuevo camino epistemológico en Latinoamérica. Abandonando los caparazones procedimentales y asumiendo miradas complejas capaces de abordar y analizar las nuevas realidades socio-educativas.

En estos desafíos epistemológicos es que construimos un modelo de análisis teórico.

Entre los debates de los pasos, los ciclos, las redes, los abordajes centrados en el estado y aquellos que impulsan otro tipo de investigación consideramos que el swarming es un modelo para comprender las realidades cada vez más complejas que los investigadores debemos abordar. 


\section{Referencias}

BALL, S. La micropolítica de la escuela. Hacia una teoría de la organización escolar. Temas de Educación. Buenos Aires: Paidós, 1989.

BALL, S. Política social y educacional, empresa social, hibridación y nuevas comunidades discursivas, Propuesta Educacional, v. 2, n. 36, Año 20 - Nov 2011, p. 25-34, 2011.

BALL, S. J. Politics and policy-makingin education. London: Routledge, 1990.

BORÓN, A., Las ciencias sociales en la era neoliberal: entre la academia y el pensamiento crítico. Tareas, Panamá (CELA - Centro de Estudios Latinoamericanos "Justo Arosemena"), n 122, enero- abril, 2006

BURCH, P. Hidden Markets: The new education privatization. Abingdon, Routledge, 2009.

BREWER, G., The Policy Sciences Emerge: To Nurture and. Structure a Discipline. Policy Sciences. Santa Monica: The. Rand Corporation, pp. 5-3, 1974

CANSINO, C. Adiós a la ciencia política - Crónica de una muerte anunciada, Metapolítica, n. 49, p. 26-37, septiembre-octubre, 2006.

CANSINO, C. La muerte de la ciencia política. Buenos Aires: Sudamericana-La Nación. 2008.

COX, C. Construcción política de reformas curriculares: el caso de chile en los noventa. Profesorado. Revista de currículum y formación del profesorado, v. 10, n. 1, p. $1-24,2006$

DALE, R. The state and education policy. Milton Keynes: Open University Press, 1989.

DE SIERRA, G., et. al Las ciencias sociales en América Latina en una mirada comparativa, In: TRINDADE, H. (org.) Las ciencias sociales en América Latina en perspectiva comparada. México, DF: Siglo XXI, pp.17-53 2006

DEUBEL, A. N. Perspectivas teóricas para el análisis de las políticas públicas: ¿de la razón científica al arte retórico?. Estudios Políticos, n. 33, p. 67-91, 2008.

DEUBEL, A. N. Políticas Públicas: Formulación, Implementación y Evaluación. Bogotá D.F: Ediciones Aurora, 2007.

DU GAY, P. Entrepreneurial governance and public management: the antibureaucrats. In: J. CLARKE et al. (eds) New Managerialism, New Welfare? London: Sage p. 62-81, 2000.

FOUCAULT, M. Of other spaces, Diacritics, v. 16, n. 1, p. 22-27, 1986.

GALINDO CÁCERES, J. Del objeto construido al objeto percibido, Estudios sobre las culturas contemporáneas. Época, v. 5, n. 9, pp. 9-24, 1999. 
JOBERT, B. ; MULLER, P. L'Etat en action. et. al. Paris: PUF, 1987

KRIESI, H. Les démocraties occidentales. Una aproche comparée. Paris: Economica. 1994.

LASSWELL, H. The Policy Orientation. In: D. Lerner; H. Lasswell, The policy sciences: recent developments in scope and method. Stanford: Stanford University Press. 1951, p. 3-15.

MAINARDES, J. Las epistemologías de la política educativa y sus contribuciones para el campo. In: TELLO, C. Epistemologías de la política educativa: posicionamientos, perspectivas y enfoques. San Pablo: Mercado de Letras.

MAINARDES, J. Análise de políticas educacionais: breves considerações teórico-metodológicas. Contrapontos, v. 9, n. 1, p. 4-16, 2009.

MENY , Y.; THOENIG, J. (1992). Las políticas públicas. Barcelona: Ariel.

MULLER, P. Les politiques publiques. Paris: PUF, 1990.

MULLER, P. (2000). L'analyse cognitive des politiques publiques: vers une sociologie politique de l'action publique. Revue Française de Science Politique, 50 (2), p. 189-208.

MULLER, P.; SUREL, Y. L'analyse des politiques publiques. Paris: Editions Montchrestien, 1998.

PANNU, R.S. Neoliberal project of globalization: prospects for democratization of education. Alberta Journal of Educational Research, v. 42, n. 2, p. 87-101, 1996.

RAAB, Ch. Theorising the governance of education. British Journal of Educational Studies, v. 42, n.1, p. 6-22, 1994.

ROBERTSON, S.L. The New Spatial Politics of (Re) Bordering and (Re) Ordering the State-Education-Citizen Relation. International Review of Education, v. 57, n. 3-4, p. 277-297, 2011.

SABATIER, P. A.; JENKINS-SMITH, H. The Advocacy coalition framework: an assessment. In: SABATIER, P. A. (Ed). Theories of the policy process. Boulder: Westview Press, p. 117-166, 1999.

SARTORI, G. ¿Hacia a dónde va la ciencia política?, Revista Política y Gobierno, v. 9, n. 2, p. 349- 354. México: CIDE, 2004.

TELLO, C. Política educativa y fraternidad en América Latina. Notas epistemológicas. In: BARRENECHE, O. (Coord.). Estudios sobre fraternidad. Buenos Aires: Editorial Ciudad Nueva, 2010. p. 187-201. (Serie Política y Sociedad). 
TELLO, C. Las epistemologías de la política educacional., Congreso Internacional educación, lenguaje y sociedad: "La educación en los nuevos escenarios socioculturales", 2., La Pampa. Actas..., Argentina, 2009.

TELLO, C. Epistemologías de la política educacional y justicia social en América Latina. Nómadas. Revista Crítica de Ciencias Sociales y Jurídicas. Monográfico América Latina Euro-Mediterranean University Institute. Universidad Complutense de Madrid. España, p. 489- 500, 2011.

TELLO, C. "Las Epistemologías de la política educacional y la concepción de producción del conocimiento 'basada en la evidencia'”. In: FERNÁNDEZ LAMARRA, N. (comp.) Estudios sobre Políticas Educativas. EDUNTREF, 2012a.

TELLO, C. Las epistemologías de la política educativa como enfoque y la vigilancia y el posicionamiento epistemológico del investigador. Práxis Educativa, v. 7, n. 1, p. 53-68, jan./jul. 2012b.

TELLO, C. "Los componentes del EEPE.", I Jornadas Latinoamericanas de Estudios Epistemológicos en Política Educacional. Conferencia Inaugural. Actas... Buenos Aires: UNTREF, 2012c.

TELLO, C. "El horizonte del Enfoque de las Epistemologías de la política educacional: la justicia social.", in: ALMEIDA, M. L y BONETTI, L. O social e as políticas educacionais na contemporaneidade: das desigualdades à violência no espaço educacional Campinas: Mercado de Letras, 2013a.

TELLO, C. Notas analíticas sobre el enfoque de las epistemologías de la política educacional, Conjectura: filosofia e educação. Universidades Caxias do Sul, 2013b.

TELLO, C. Las epistemologías de la política educacional. Notas históricas y epistemológicas sobre el campo. In: TELLO, C. (org) Las epistemologías de la política educacional. Enfoques y Perspectivas para el análisis de Políticas educacionales. Campinas. SP: Mercado de Letras. 2013c.

TELLO, C. El campo teórico de la política educacional: modelos, abordajes y objetos de estudio. Jornal de Políticas Educacionais. UFPR. En prensa. 2014a.

TELLO, C. (2014) The theoretical field of education policy: characteristics, objects of study and mediations A Latin American perspective. American Journal of Educational Research. En prensa. 2014b.

TELLO, C.; MAINARDES, J. La posición epistemológica de los investigadores en Política Educativa: debates teóricos en torno a las perspectivas neomarxista, pluralista y pos-estructuralista. Archivos Analíticos de Políticas Educativas, v. 20, n. 9, p. 1 -33, 2012, Disponible en: <http://epaa.asu.edu/ojs/article/view/988>. Acceso: 24 mar. 2012. 
TELLO, C; MAINARDES, J. El pluralismo como perspectiva epistemológica en la investigación en política educativa. Reflexiones desde el enfoque de las Epistemologías de la política educativa, 2014. Mimeo (inédito)

UNESCO, International Conference on: Methods in Political Science, 13 September 1948-16 September 1948. Statement issued by the members of the Conference, 16 September 1948. Paris, UNESCO, 28 April 1949.

VERGARA ESTEVEZ, J. "Las ciencias sociales latinoamericanas: desarrollo, crisis y perspectivas.", Seminario Internacional de la Comisión de Epistemología y Política de CLACSO, Santiago de Chile, 1991.

VIRNO, P. Gramática de la multitud. Para un análisis de las formas de vida contemporáneas. Buenos Aires: Colihue, 2003.

WALLERSTEIN, I. Impensar las ciencias sociales. México: Siglo XXI, 1999.

Recebido em: 23/09/2015

Aprovado em: 20/10/2015 